SCIENTIFIC LETTER

\title{
Obtaining informed consent from patients in the early phase of acute myocardial infarction: physicians' experiences and attitudes
}

\author{
A Ågård, J Herlitz, G Hermerén
}

Heart 2004;90:208-210. doi: 10.1136/hrt.2003.021501

l: is possible to question whether patients suffering from the early phase of an acute myocardial infarction (AMI) are able to give free and informed consent and whether or not it is ethically defensible to include them-those patients suffering from more severe symptoms, in particular-in research projects. ${ }^{1}$ In fact, it has been shown that a reasonable number of patients who have given their consent in this situation are only able to recall very little of the information they received about the study and that only a minority read through the consent form before signing it. ${ }^{2-4}$

The aim of this study is to determine how physicians, who have been involved in including patients in intervention trials in the early phase of their AMIs, experienced the informed consent procedures. In our view, data obtained from those people who are actually confronted by the research subjects and who are responsible for carrying out the consent procedure should also be taken into account, when the most appropriate ways of including these patients in studies are discussed. To our knowledge no survey of physicians relating to this issue has previously been undertaken.

\section{METHODS}

A questionnaire containing questions relating to the informed consent procedure in the context of AMI was distributed to all 1002 physicians registered as members of the Swedish Society of Cardiology in 2001. In all, 739 questionnaires were returned, giving a response rate of $74 \%$. Those without experiences of including AMI patients in trials were excluded $(\mathrm{n}=174)$. Questionnaires were also excluded, if more than two questions were incorrectly filled in $(n=21)$. The results are based on an analysis of the remaining 544 questionnaires.

Statistical analyses were performed in order to determine whether the respondent's sex, their experiences of including patients in trials during the last year, and the setting in which they were working influenced their way of answering.

The respondents were able to comment on all the items, making it possible for them to clarify their answers or to provide some additional information. A total of 881 comments were distributed between the nine questions, ranging between 58-149 for each individual question.

\section{RESULTS}

The main results from the survey are presented (table 1). Two thirds $(68 \%)$ of the physicians felt that too much information had to be given to the potential research subject suffering from an AMI. Most of the physicians (86\%) felt that the patients were not generally able to understand more than some parts of the information given to them. Approximately one in three respondents estimated that the patients in question read through the consent form in some detail.
Fifty six per cent felt that it should also be compulsory to provide written information in the acute situation. Male doctors were more inclined to prefer only to provide verbal information compared with their female counterparts (question 7: $45 \% v 33 \%, \mathrm{p}<0.01$ ). Half the male physicians felt that the need to obtain informed consent could be abandoned when the patients were unable to understand information, whereas only a third of their female colleagues felt the same (question 8: $50 \%$ v 32\%, p < 0.01).

Most of the respondents had not experienced patients reacting negatively to being asked to participate in a trial, at least not to any great extent. Fifteen per cent felt that the patients' trust in the physicians was affected negatively by the process of obtaining informed consent, whereas 34\% actually felt the contrary.

It was apparent from the written comments that the physicians act in different ways when they obtain informed consent. Moreover, the large variation among patients, for example, when it came to their ability to understand information, was emphasised. One common criticism focused on the need to obtain the patients' signature on paper during the acute phase of the disease. Some stressed that the understanding of the potential research subjects and their willingness to participate in a study are to some extent dependent on how the physician acts.

\section{DISCUSSION}

One major methodological problem, which has to be taken into consideration when analysing the results, is the fact that the questions were generally formulated. The respondent's answers therefore had to be based on a rough estimation of the average situation when it came to their experience of including a variety of patients in trials of different kinds.

The physicians in this survey felt that that the amount of information that was to be given about the trials was excessive, mainly because most patients suffering from an AMI are not regarded as being capable of reaching more than a limited level of understanding in this situation. Even though physicians are expected to follow the instructions given in the consent form and to apply them generally, there appears to be a great deal of variation among physicians when it comes to the way they actually obtain informed consent. Perhaps the best approach is to assume the patient has been given clear and brief information about some essential aspects of the study and then to ask for verbal consent. ${ }^{3}$ To minimise the number of patients who find this request unpleasant, physicians should consider not only whether they should ask but also how to behave when doing so.

The complexity of the question of whether or not it should it be possible to include a patient with AMI, whose capacity to understand information is highly limited, is illustrated by the different opinions expressed by the respondents. Some of 
Table 1 The questionnaire and numeric results

1. What is your estimation of how much of the written information the patient reads before they sign the consen form?

None/almost none $\quad 30 \%(159)$

Some $\quad 36 \%(189)$

All/almost all $\quad 30 \%(158)$

Uncertain $4 \%(24)$

2. How much of the information received by the patient, in this acute phase of the myocardial infarction, do you think they understand?

None/almost none $\quad 24 \%(130)$

$62 \%(329)$

All/almost all $\quad 14 \%(74)$

3. Do you feel that the patient reaches such a level of understanding, that it is possible to claim that they really know what they are accepting or refusing?

Always/most often 34\% (183)

\begin{tabular}{ll} 
Sometimes & $38 \%(205)$ \\
\hline
\end{tabular}

Never/almost never $\quad 25 \%(133)$

Uncertain $3 \%(17)$

4. How do patients react when they are asked, during the acute phase of a myocardial infarction, if they are willing to participate in a trial?

Very/somewhat negatively $27 \%(143)$

Neither negatively nor positively $25 \%(132)$

Very/somewhat positively $39 \%(210)$

Uncertain $9 \%(48)$

5. In what way do you think the patients' trust in the physicians is affected by being asked, during the acute phase of a myocardial infarction, if they are willing to participate in a trial?

Very/somewhat negatively $15 \%(82)$

Neither negatively nor positively $\quad 45 \%(239)$

Very/somewhat positively $34 \%(183)$

Uncertain 6\% (32)

6. What is your opinion about the amount of information you are supposed to give to the patient at this point in time?

Far too incomplete/somewhat incomplete $\quad 5 \%(29)$

Adequate $23 \%(120)$

Far too comprehensive/somewhat comprehensive $\quad 68 \%(359)$

Uncertain $4 \%(21)$

7. Which of the following alternatives do you consider to be appropriate for the inclusion of patients with acute myocardial infarctions in a trial?

Verbal + witten consent

Verbal

$54 \%(292)$

$41 \%(220)$

Uncertain $3 \%(18)$

8. Taking the facts below into consideration, do you feel that it should be possible to include these patients in trials?

Yes $37 \%(197)$

No $44 \%(235)$

Uncertain $\quad 19 \%(104)$

9. Would you accept being included in an interventional trial in the acute phase of a myocardial infarction without being asked?

$\begin{array}{lc}\text { Yes } & 20 \%(108) \\ \text { No } & 71 \%(378) \\ \text { Uncertain } & 9 \%(46)\end{array}$

In order to include a patient who is suffering from $\mathrm{AMI}$ in a trial, they must first be given adequate information about the study and then give their consent to participate, both verbally and in writing. Some patients with AMI are strongly affected by the condition and so may have difficulty understanding the information they receive or even completely misunderstand.

them emphasised the principle of respecting the patients' autonomy, whereas others put the medical benefits, which could be achieved by permitting research, first. The latest version of the Declaration of Helsinki states that research on groups with reduced autonomy is permitted "if the physical/ mental condition that prevents obtaining informed consent is a necessary characteristic of the research population". Moreover, if certain conditions are satisfied, it is said that research can be initiated even though informed consent has not been obtained instantly (paragraphs 24-26). ${ }^{5}$ It can be claimed that patients who are severely impaired by an AMI represent "a necessary characteristic of the research population". As some respondents pointed out, the benefits of a new treatment might actually be greatest in those patients who are most affected by the disease.
We feel that it is important for the international societies of cardiology to develop more specific instructions for dealing with the need to obtain informed consent when patients are suffering from acute cardiac conditions, which most often and to a varying degree influence their autonomous capacity.

\section{Authors' affiliations}

A Ågård", Department of Medical Ethics, Lund University, Lund, Sweden J Herlitz, Department of Cardiology, Sahlgrens University Hospital, Gothenburg, Sweden

G Hermerén, Department of Medical Ethics, Lund University

*Also Department of Medicine, Sahlgrens University Hospital, Gothenburg, Sweden 
Correspondence to: Dr Anders Ågård, Department of Medicine, Sahlgrens University Hospital, SE-413 45 Göteborg, Sweden; anders.s.agard@vgregion.se

Accepted 25 September 2003

\section{REFERENCES}

1 Grim PS, Singer PA, Gramelspacher GP, et al. Informed consent in emergency research. Prehospital thrombolytic therapy for acute myocardial infarction. JAMA 1989:262:252-5.
2 Yuval R, Halon DA, Merdler A, et al. Patient comprehension and reaction to participating in a double-blind randomized clinical trial (ISIS-4) in acute myocardial infarction. Arch Intern Med 2000;160:1142-6.

3 Ågård A, Hermerén G, Herlitz J. Patients' experiences of intervention trials on the treatment of myocardial infarction: is it time to adjust the informed consent procedure to the patient's capacity? Heart 2001;86:632-7.

4 Williams BF, French JK, White HD. Informed consent during the clinical emergency of acute myocardial infarction (HERO-2 consent substudy): a prospective observational study. Lancet 2003:361:918-22.

5 World Medical Assembly Declaration of Helsinki. Ethical principles for medical research involving human subjects. JAMA $2000 \cdot 284: 3043-5$

\section{IMAGES IN CARDIOLOGY}

\section{Novel imaging techniques for the differentiation of a left ventricular mass}

A 39 year old man presented with a right popliteal artery embolism and was successfully treated with open embolectomy. He was a heavy smoker but had no history of thrombosis, peripheral vascular disease, arrhythmias, ischaemic heart disease, or chest pain. He denied any drug use and his total cholesterol was $5.3 \mathrm{mmol} / \mathrm{l}$. The ECG showed inferolateral Q waves and $\mathrm{T}$ wave inversion. Transthoracic echocardiography showed infero-apical akinesia and an apical left ventricular mass (upper panel, middle column). Contrast echocardiography using a low power technique showed reduced enhancement in the infero-apical region in keeping with myocardial infarction and no enhancement of the mass (lower panel, middle column). Contrast enhanced cardiac magnetic resonance also demonstrated a lack of uptake in the mass on turbo spin-echo imaging (upper panel, right column), and on delayed imaging with an inversion recovery sequence, hyperenhancement of the infero-apical region was visible, confirming infarction (lower panel, right column). Coronary angiography revealed smooth unobstructed epicardial vessels. Both contrast echocardiography and magnetic resonance techniques suggested the mass was thrombus rather than tumour and the patient received warfarin treatment for six weeks. Repeat echocardiography
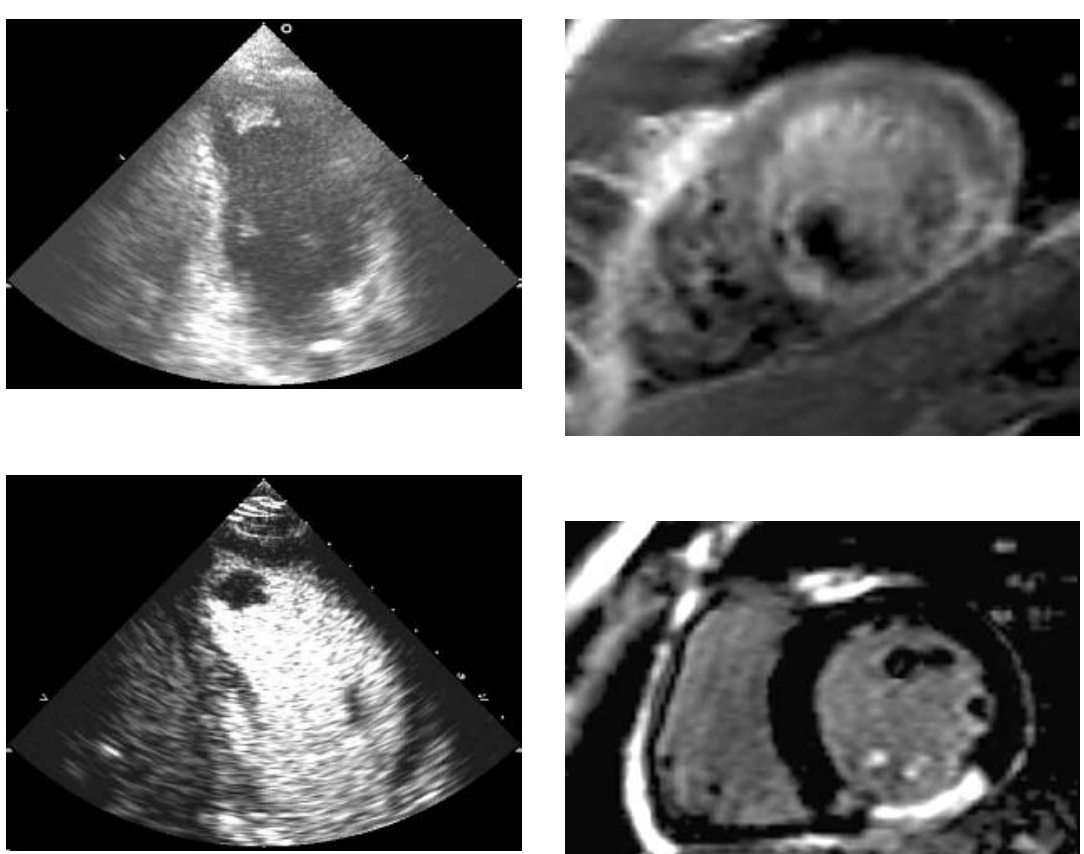

showed complete resolution of the mass.

The differentiation of intracardiac thrombus from tumour is important and these images demonstrate the ability of these two non-invasive techniques to assess accurately the nature of the mass in this case. The cause of the myocardial infarction was presumed to be a thrombosis on a non-obstructive

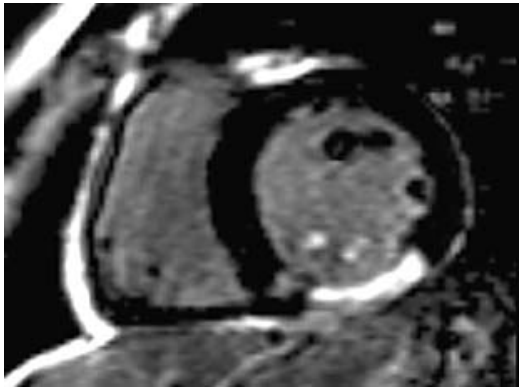

coronary plaque that subsequently recanalised, though the lack of chest pain and mode of presentation are unusual, particularly in such a young man.

J Timperley

S G Myerson

A R J Mitchell mitcharj@doctors.org.uk 